\title{
Representações Sociais de Idosos Institucionalizados Acerca da Promoção da Saúde Por Meio de Ações Dialógicas
}

\author{
Telma Pelaes de Carvalho, ${ }^{1}$ Mariluci Hautsch Willig, ${ }^{2}$ Luciana Puchalski Kalinke, ${ }^{2}$ \\ Nadine Biagi Sousa Ziesemer, ${ }^{3}$ Ana Paula Berberian, ${ }^{2}$ Giselle Aparecida Athayde Massi ${ }^{2}$
}

\begin{abstract}
RESUMO
Objetivo: Conhecer as representações sociais de idosos institucionalizados acerca de atividades de promoção da saúde, mediadas por ações dialógicas. Método: estudo qualitativo descritivo, ancorado na Teoria das Representações Sociais e organizado em três etapas. Na primeira aplicou-se um questionário estruturado para caracterizar os participantes da pesquisa, na segunda etapa foram realizados grupos de promoção da saúde e, na terceira, foram feitas entrevistas individualizadas, cujas respostas foram processadas pelo software Iramuteq. Resultados: Participaram do estudo 14 pessoas com mais de 60 anos. Um do sexo masculino e 13 do sexo feminino. A idade média foi de 77 anos, a escolaridade predominante foi o Ensino Fundamental incompleto e o tempo de residência nas Ilpis variou de 1 a 9 anos. O software concentrou as respostas dos idosos das entrevistas em três classes, assim nominadas: 1) Conversar para conhecer as outras pessoas, conviver com elas e me fortalecer; 2) Participar do encontro ajuda a enfrentar a vida institucionalizada; 3) Nunca é tarde para cuidar da saúde. Considerações finais: os enunciados elaborados pelos participantes revelam que a interação social promove a produção e a troca de conhecimento, o fortalecimento de relações interpessoais, o compartilhamento de experiências vividas e o estabelecimento de confiança mútua. As atividades mostraram-se eficazes para os idosos, ajudando-os a (re)significar a vida institucionalizada.
\end{abstract}

Palavras-chave: Representação social. Idosos. Institucionalização. Promoção da saúde. Ações dialógicas.

\section{SOCIAL REPRESENTATIONS OF INSTITUTIONALIZED ELDERLY PEOPLE} ABOUT HEALTH PROMOTION THROUGH DIALOGIC ACTIONS

\begin{abstract}
Objective: To know the social representations of institutionalized elderly people about health promotion activities, mediated by dialogic actions. Method: Qualitative and descriptive study, underpinned by the Social Representation Theory and organized in three steps. In the first, we applied a structured questionnaire to characterize the research participants; in the second step, we organized health promotion groups; and, in the third, we conducted individualized interviews, whose responses were processed by the Iramuteq software. Results: The study was attended by 14 people older than 60 years of age. Participants were 01 male and 13 female. The average age was 77 years, the predominant schooling was incomplete elementary school and the time of residence in Long-Term Institutions for the Elderly (LTIEs) ranged from 1 to 9 years. The software concentrated the responses of the elderly people to the interviews in three classes, namely: 1) To talk to each other to meet other people, live with them and strengthen myself; 2) To participate in the meetings helps us to deal with institutionalized life; 3 ) It is never too late to take care of our health. Final considerations: The statements made by the study participants reveal that social interaction promotes the production and exchange of knowledge, the strengthening of interpersonal relationships, the sharing of lived experiences and the establishment of mutual trust. The activities proved to be effective for the elderly citizens, helping them to (re) define institutionalized life.
\end{abstract}

Keywords: Social representation. Elderly. Institutionalized. Health Promotion. Dialogic actions.

RECEBIDO EM: 27/2/2020

MODIFICAÇÕES SOLICITADAS EM: 1/6/2020

ACEITO EM: 4/6/2020

\footnotetext{
Autora correspondente. Instituto Federal do Paraná (IFPR). Rua João Negrão, n. 1285, Bairro Rebouças, Curitiba/PR, Brasil, CEP 80.230-150. http://lattes. cnpq.br/1926420088217255. https://orcid.org/0000-0002-2912-2111. telmacarvalho2006@gmail.com

2 Universidade Federal do Paraná - UFPR. Curitiba/PR, Brasil.

${ }^{3}$ Instituto Federal do Paraná - IFPR. Curitiba/PR, Brasil.
} 


\section{INTRODUÇÃO}

As populações estão envelhecendo rapidamente e estima-se que, em 2050, o número de idosos aumentará de 600 milhões para aproximadamente dois bilhões em todo o mundo, e tal crescimento será mais rápido em países emergentes (OMS, 2016). Esse aumento já é legitimado no Brasil desde a década de 60 do século 20 , e, em 2017, foram contabilizados 30,2 milhões de idosos em todo o país, representando $13 \%$ da população brasileira (MINAYO; GUALHANO, 2017).

Especificamente na região Sul, registra-se o maior percentual de idosos, perfazendo $15,2 \%$ da população. Com esse contingente populacional já é possível identificar a ampliação da expectativa de vida, agregando o aumento de forma semelhante à demanda por Instituições de Longa Permanência para Idosos (Ilpi), na medida em que é expressiva a quantidade de brasileiros e brasileiras, com mais de 60 anos, que precisam de cuidados especializados (BRASIL, 2018; IBGE, 2016).

Embora o Estatuto do Idoso recomende a permanência da pessoa idosa no seio familiar, quando a família não pode lhe dar suporte (BRASIL, 2003) e o próprio idoso não tem condições para contratar serviços de um cuidador particular, a institucionalização torna-se a única opção. Cabe explicitar que, segundo o Ministério de Desenvolvimento Social, o número de idosos institucionalizados cresceu 33\% entre 2012 e 2017, passando de 45.827 para 60.939 (BRASIL, 2018). Com relação às Ilpis, convém ressaltar a imprescindibilidade de as mesmas contarem com profissionais de saúde preparados para cuidar dos idosos, proporcionando um atendimento personalizado, capaz de preservar a autonomia e a independência da pessoa idosa, respeitando a manutenção dos seus papéis sociais (BRASIL, 2003; BARROS et al., 2016 ), pois, a promoção da autonomia e a integração social da pessoa idosa, independentemente de ela residir sozinha, com sua família ou em uma Ilpi, são direitos assegurados por dispositivos legais que constam da Política Nacional do Idoso (BRASIL, 1994).

Nessa trilha de políticas públicas voltadas à população que envelhece, o Estatuto do Idoso, pautado na perspectiva dos direitos sociais de pessoas com mais de 60 anos, estabelece obrigações legais a serem observadas pelas Ilpis, as quais devem direcionar ações voltadas ao atendimento personalizado e em pequenos grupos de residentes, envolvendo a participação do idoso em atividades comunitárias (BRASIL, 2003). Mais especificamente, a Política Nacional de Saúde da Pessoa Idosa anuncia que a compreensão da saúde dos idosos, além da preocupação com a presença ou a ausência de doenças orgânicas, está atrelada à sua capacidade de tomar decisões relacionadas à vida cotidiana (BRASIL, 2006). Essa política, voltada à otimização de oportunidades de participação social, bem como à manutenção, recuperação e promoção da autonomia da pessoa idosa, cita a relevância da promoção da saúde em todo o curso de vida, indicando que a interação social e o diálogo com idosos devem ganhar força em todos os espaços sociais ocupados pelos mais velhos.

Dessa forma, trabalhos voltados à interação social e ao diálogo devem ser preponderantemente considerados nas Ilpis, na medida em que os profissionais da saúde, bem como pesquisadores envolvidos com essa temática, além de voltarem-se aos cuidados com a saúde física dos residentes, precisam ampliar as possibilidades de promover a participação de idosos em redes comunitárias e familiares, as quais oportunizam a manutenção dos seus vínculos afetivos e de seus papéis como beneficiários e contribuintes da comunidade em que estão inseridos (SIMEAO et al., 2018; ANDRADE et al., 2018).

Para sustentar essa atividade foi utilizado o norteador teórico alicerçado na Teoria das Representações Sociais, que visa a compreender os universos consensuais, expressos nas relações cotidianas que compõem o senso comum, orientando os indivíduos quanto aos modos de compreender o seu meio social e de atuar nele (MOSCOVICl, 2012).

Com o esteio das relações dialógicas, os indivíduos ampliam suas possibilidades de representar o mundo que o cerca e tornam-se mais expressivos na medida em que são valorizados como sujeitos sociais, interpretando a realidade cotidiana, conforme propõe a Teoria das Representações Sociais (NASCIMENTO, 2016).

Em razão do aumento significativo na demanda por cuidados prestados por Instituições de Longa Permanência, este estudo objetiva conhecer as representações sociais de idosos institucionalizados acerca de atividades de promoção da saúde, mediadas por ações dialógicas.

\section{MÉTODO}

Trata-se de uma pesquisa qualitativa descritiva, ancorada na Teoria das Representações Sociais (TRS), e obedecendo aos princípios da Resolução no 466 de 12 de dezembro de 2012 do Conselho Nacional de Saúde (BRASIL, 2012). A pesquisa recebeu aprovação do Comitê de Ética em Pesquisa da Sociedade 
Evangélica Beneficente de Curitiba - Paraná -, Brasil, sob o CAAE no 39125514.5.0000.0103 e Parecer no 908.910/2014, em 8 de dezembro de 2014.

\section{Participantes do estudo}

Como forma de preservar o anonimato dos participantes, optou-se por substituir os nomes dos 14 idosos institucionalizados pelo numeral arábico de $1 \mathrm{a}$ 8 para os participantes da Ilpi "Aiyra" e para os participantes da Ilpi "Belet" o numeral arábico de 9 a 14. 0 estudo foi realizado em duas Ilpis, que são reconhecidas pelos nomes fictícios Aiyra e Belet.

\section{Cenários do estudo}

- A Instituição de Longa Permanência (Ilpi) Aiyra é uma instituição privada e administrada por uma congregação religiosa. Ela atende idosos de ambos os sexos, independentes e semi-independentes, que contribuem com mensalidades relativas aos serviços prestados. Nesta Ilpi atuam um clínico geral, fisioterapeutas, técnicas e auxiliares de enfermagem, nutricionista, cozinheiras, copeiras e cuidadores de idosos.

- A Instituição de Longa Permanência (Ilpi) Belet é uma associação mantida pelo poder público e por meio de donativos da comunidade e de empresas particulares locais, abrigando exclusivamente mulheres com idade igual ou superior a 60 anos. As moradoras que têm alguma renda pagam mensalidades, as quais correspondem a $70 \%$ do salário mínimo vigente no território nacional. Nessa Ilpi a assistência é realizada por técnicas e auxiliares de enfermagem, cuidadores de idosos, nutricionista, cozinheiras, educador físico e voluntários.

\section{Critérios de elegibilidade para o estudo}

Participaram da pesquisa residentes de ambas as Ilpis, com 60 anos ou mais, que tivessem interesse em integrar atividades dialógicas e que respondessem favoravelmente o Miniexame do Estado Mental (Meem) (MARTINS et al., 2019) estabelecido, de acordo com a escolaridade.

\section{Critérios de exclusão para o estudo}

Foram excluídos do estudo pessoas com menos de 60 anos e que tivessem dificuldades para falar ou para compreender e participar de situações dialógicas grupais.
Nesse sentido, cabe ressaltar que, de um total de 93 idosos residentes na Ilpi Aiyra, apenas 8 puderam participar, pois atendiam aos critérios de inclusão. Na Ilpi Belet, das 28 idosas institucionalizadas apenas 6 tiverem seus desempenhos satisfatórios, de acordo com os mesmos critérios de inclusão.

\section{Coleta e registros das informações}

\section{Primeira etapa}

A primeira etapa concentrou-se na realização de convites e esclarecimentos acerca da pesquisa e seus objetivos. Para aqueles que aceitaram participar da pesquisa, foi compartilhado e assinado o Termo de Consentimento Livre e Esclarecido (TCLE). Na sequência, foram aplicados o questionário estruturado para caracterizar os participantes da pesquisa, bem como o Miniexame do Estado Mental (Meem).

\section{Segunda etapa}

Na segunda etapa foram realizados 16 encontros dialógicos grupais de promoção à saúde em cada instituição. Esses aconteceram semanalmente, em horários predeterminados pela direção administrativa das Ilpis, realizados em uma sala reservada que permitiu seu desenvolvimento em um ambiente resguardado pela privacidade, envolvendo a pesquisadora e os participantes do estudo.

Cada encontro teve a duração de, aproximadamente, 90 minutos. Os assuntos discutidos foram escolhidos pelo grupo a partir das opções oferecidas pela pesquisadora, sendo utilizados textos variados que discorriam sobre aspectos relacionados à promoção da qualidade de vida, participação social, autonomia, autocuidado, bem como políticas públicas e dispositivos legais que amparam as pessoas idosas.

\section{Terceira etapa}

A terceira etapa consistiu na realização de entrevistas semiestruturadas, a partir das quais foram coletados relatos dos participantes a fim de conhecer as representações sociais de idosos institucionalizados acerca das atividades desenvolvidas no grupo de promoção à saúde.

As entrevistas prolongaram-se entre 20 e $40 \mathrm{mi}$ nutos, e, ao seu término, foram transcritas e validadas pelos participantes, quando puderam tomar ciência das suas produções discursivas, realizando modificações que julgassem necessárias.

Ressalta-se que, nas entrevistas, os idosos faziam referências ao trabalho dialógico de grupos de promoção da saúde, utilizando-se das palavras pales- 
tra, reunião, pesquisa, consulta e encontro. Dessa for$\mathrm{ma}$, buscou-se unificar as palavras mencionadas pelos idosos para encontro, como forma de sinônimos para auxiliar o processamento dos dados pelo software do material coletado.

\section{Processamento e Análise das Informações}

Após a finalização da coleta de dados os relatos dos idosos foram submetidos ao software Iramuteq (Interface de R pourles Analyses Multidimensionnelles de Textes et de Questionnaires), desenvolvido por Pierre Ratinaud, que permite realizar análises estatísticas sobre corpus textuais (CAMARGO; JUSTO, 2013).

A partir do uso desse software, foi possível fazer a Classificação Hierárquica Descendente (CHD), obtendo-se classes de segmentos de texto que apresentam vocabulário semelhante entre si.

Especificamente nas pesquisas sustentadas pelas representações sociais, este tipo de análise permite organizar as manifestações linguísticas e indicar conhecimentos do senso comum sobre um dado objeto ou fenômeno, interpretados a partir de campos lexicais e de contextos semânticos.

Cabe esclarecer que os dados processados pelo Iramuteq são considerados válidos apenas quando apresentarem uma retenção de, no mínimo, 75\% dos segmentos textuais (CAMARGO; JUSTO, 2013). Neste estudo foram registrados $82,77 \%$ de aproveitamento dos textos, sendo possível alcançar adequadamente a validação do processamento dos dados.

\section{RESULTADOS}

O perfil societário, referente a sexo, idade, estado civil, escolaridade, ocupação anterior e tempo de institucionalização, são apresentados no Quadro 1.

Quadro 1 - Caracterização dos participantes do estudo

\begin{tabular}{|l|l|l|}
\hline CARACTERÍSTICAS & \multicolumn{1}{|c|}{ ILPI AIYRA } & \multicolumn{1}{c|}{ ILPI BELET } \\
\hline Sexo & $\begin{array}{l}\text { Mulheres 7 } \\
\text { Homens 1 }\end{array}$ & Mulheres 6 \\
\hline Idade & 60 a 92 anos & 70 a 86 anos \\
\hline Estado civil & $\begin{array}{l}\text { Casada 1 } \\
\text { Solteiro 2 } \\
\text { Viúvas 4 } \\
\text { Divorciada 1 }\end{array}$ & $\begin{array}{l}\text { Casada 1 } \\
\text { Solteiro 2 } \\
\text { Viúvas 2 } \\
\text { Divorciada 1 }\end{array}$ \\
\hline Escolaridade & $\begin{array}{l}\text { Ensino Fundamental } \\
\text { completo 4 } \\
\text { Ensino Fundamental } \\
\text { incompleto 3 } \\
\text { Ensino Superior } \\
\text { completo 1 }\end{array}$ & $\begin{array}{l}\text { Ensino Fundamental } \\
\text { completo 2 } \\
\text { Ensino Fundamental } \\
\text { incompleto 4 }\end{array}$ \\
\hline
\end{tabular}

\begin{tabular}{|l|l|l|}
\hline Ocupações & Dona de casa 3 & Dona de casa \\
& Bordadeira & Cantora de forró \\
& Diarista & Cozinheira \\
& Doméstica & Atendente de \\
& Escrevente de & enfermagem \\
& cartório & Professora \\
& Maestrina & Doméstica \\
\hline $\begin{array}{l}\text { Tempo de } \\
\text { residência na Ilpi }\end{array}$ & 1 a 9 anos & 1 a 6 anos \\
\hline
\end{tabular}

Fonte: as autoras.

Os participantes são majoritariamente mulheres (13) com idade média de 77 anos; 6 viúvas; 6 com Ensino Fundamental completo; a ocupação anterior de 4 delas era dona de casa. $O$ tempo de residência nas Ilpis variou de 1 a 9 anos. Das entrevistas realizadas com os 14 participantes, o software Iramuteq elaborou a Classificação Hierárquica Descendente, que permitiu organizar as manifestações linguísticas, as quais concentraram, em termos percentuais, três classes de segmentos textuais, explicitadas no Quadro 2.

Quadro 2 - Nominação das classes representadas

\begin{tabular}{|c|l|c|}
\hline $\begin{array}{c}\text { Número } \\
\text { das classes }\end{array}$ & \multicolumn{1}{|c|}{$\begin{array}{c}\text { Temática das classes apresentadas } \\
\text { pelos idosos }\end{array}$} & $\%$ \\
\hline 1 & $\begin{array}{l}\text { Conversar para conhecer as outras } \\
\text { pessoas conviver com elas e me } \\
\text { fortalecer }\end{array}$ & 53,13 \\
\hline 2 & $\begin{array}{l}\text { Participar do encontro ajuda a enfrentar } \\
\text { a vida institucionalizada }\end{array}$ & 23,75 \\
\hline 3 & Nunca é tarde para cuidar da saúde & 23,12 \\
\hline \multicolumn{2}{|l}{} & Total 100 \\
\hline
\end{tabular}

Fonte: as autoras.

\section{Classe 1}

Na classe 1, a temática revela que conversar para conhecer as outras pessoas, conviver com elas e me fortalecer, constituiu a maior compreensão representacional encontrada nessa pesquisa, com 53,13\%, enaltecendo a importância das trocas mediadas pela linguagem entre os idosos institucionalizados. Nessa classe são apresentadas as representações sociais dos participantes a respeito da afinidade e da importância que as conversas significaram para eles ao longo da realização dos grupos de promoção à saúde, como asseveram os participantes:

"Escutar as histórias me ajuda a ficar bem e a conhecer quem elas eram; teve dias que eu estava desanimada aqui em casa e com os encontros ficava mais animada" (P2).

"Agora depois dos encontros conheço mais as pessoas da casa e ficamos mais juntas. De algumas sabia só o nome. Fui conhecendo depois que vim participar dos encontros." (P4) 
Depois que eu comecei com o grupo de vocês senti muita diferença. Me sinto melhor; agora converso com todos, gosto dos encontros, foi muito bom. Favoreceu a minha convivência com todos em geral" (P5).

"Os encontros me trouxe mais satisfação e mais força pra viver. Pode ter certeza. Foi bom conversar e participar" (P6).

"Me senti melhor e mais animada com os encontros. As nossas conversas foram boas, isso sim" (P11).

\section{Classe 2}

Na classe temática 2, participar dos encontros ajuda a enfrentar a vida institucionalizada representou $23,75 \%$ dos resultados. Os enunciados, nessa classe, revelam que, ao participarem dos grupos de promoção da saúde, os idosos integrantes da pesquisa indicam a relevância de atividades dialógicas, conforme os relatos apresentados na sequência:

"Eu gostaria que continuasse, não ficaria cansativo, não. Aqui tudo é no mesmo horário, tudo igual" (P1).

"E escutar as histórias me ajuda a ficar bem e menos desanimada. Todos aqui falam que gostam de participar, podia continuar, estávamos precisando participar de uma coisa assim aqui da casa. Isso me marcou, vai dar saudade" (P2).

"Vi que a cada vez tinha coisas boas e melhores. Aqui a gente fica muito sozinha e parada. Eu ia preenchendo um vazio em mim" (P4).

\section{Classe 3}

A classe 3 , definida como nunca é tarde para cuidar da saúde, representou $23,12 \%$ das manifestações linguísticas elaboradas pelos idosos participantes do grupo de promoção à saúde. Tal classe evidencia que a atividade dialógica desenvolvida com e pelo grupo viabilizou a compreensão representacional sobre a relevância de considerar a própria saúde e o autocuidado no ambiente institucionalizado. Para explicitar essa compreensão, os participantes produziram os textos que seguem:

"Fez um bem para mim participar dos encontros. Agora eu procuro me cuidar, eu estou fazendo mais pela minha saúde" (Р3).

"Agora vou me cuidar muito mais" (P7).

"Foi bom ter vocês falando de saúde, ajuda a gente a se animar e se cuidar" (P8).

\section{DISCUSSÃO}

O apanhado que será apresentado na discussão deste estudo, é que conversar para conhecer as outras pessoas residentes nas Ilpis, participar do encontro ajuda a enfrentar a vida institucionalizada e que nunca é tarde para cuidar da saúde, são representações sociais que os idosos, participantes desta pesquisa, desenvolveram a partir de atividades mediadas por ações dialógicas.

Para eles, práticas interlocutivas, baseadas nos preceitos da promoção de saúde, assumem papel preponderante para que o convívio, a participação e o autocuidado sejam estabelecidos no espaço físico e social das Ilpis. Para analisar os resultados deste estudo, na sequência são apresentadas discussões referentes às classes temáticas 1 , 2 e 3 .

\section{Classe 1}

Na classe 1, "conversar para conhecer as outras pessoas, conviver com elas e me fortalecer" é uma representação social fortemente marcada no discurso produzido pelos participantes, a qual indica a relevância de as Ilpis desenvolverem atividades pautadas em processos interlocutivos para ajudar os idosos que residem no mesmo espaço a se conhecerem, melhorando a convivência entre eles e fortalecendo vínculos. Outras pesquisas evidenciam essa questão, indicando que dialogar incita e melhora a convivência de pessoas que residem no mesmo local e também ajuda os idosos a ampliar sua participação social (YARCE-PINZÓN; HIDALGO-SOTELO; NARVÁEZ-CEBALLOS, 2018).

O diálogo confere esteio para que pessoas idosas continuem a viver dignamente, superando suas próprias percepções e suposições de que a velhice está vinculada apenas aos estereótipos negativos, representados na maior parte das sociedades ocidentais. Além disso, mais especificamente no que se refere ao ato de conversar para conhecer as outras pessoas, estudos já reconhecem a importância de trocas mediadas pela linguagem e a promoção do convívio social como essenciais para os idosos manterem a saúde física e mental (WHO, 2015).

Ao dialogarem, os idosos estabelecem e fortalecem vínculos, atenuam o isolamento, consolidam as relações sociais e alcançam melhores resultados no que se refere à promoção da própria qualidade de vida (ALVES, 2016). Além de suscitar a criação de vínculos de amizade e confiança, é gratificante para o idoso conversar sobre suas experiências, revigorando e fortalecendo a sua narrativa (BOSI, 2008). Essa narrativa, por sua vez, recompõe as histórias de vida das 
pessoas idosas, atualizando as representações sociais que estabelecem para compreender os fenômenos do mundo em que se inserem (BOSI, 2003).

Entre os direitos fundamentais da pessoa idosa está a importância da ampliação de sua rede de contato e do seu protagonismo social, bem como de sua integração nas atividades desenvolvidas pela comunidade local (WHO, 2005a). Estudos mostram que idosos inativos, que não participam de atividades coletivas, tendem a apresentar maior declínio da saúde física e mental, com perda de capacidades funcionais, com enfraquecimento de laços familiares e sociais (MAYORALAS et al., 2015).

Dessa forma, destaca-se a relevância de as IIpis desenvolverem atividades de promoção da saúde mediadas dialogicamente, facilitando a ampliação de redes sociais de seus residentes e favorecendo a adaptação deles à realidade institucionalizada em que se encontram, pois, além de ser uma prática diferente para a vida cotidiana dos idosos, as atividades dialógicas, ancoradas na teoria da representação social, configuram-se como estratégias capazes de proporcionar-lhe convívio, (re)conhecimento do outro e de si próprio, ânimo, lazer, diversão, novidades e ampliação de redes sociais (MAYORALAS et al., 2015; MANTOVANI, LUCCA; NERI, 2016).

\section{Classe 2}

Os enunciados apresentados na classe 2 revelam que a participação no encontro ajudou os idosos a enfrentarem a vida institucionalizada, pois sentem-se instigados, interessados em saber dos assuntos a serem tratados a cada encontro, e valorizados ao serem convidados a relatar suas experiências. Essas representações sociais, estabelecidas e expandidas mediante a interação entre as pessoas, parecem encorajar os participantes a integrarem-se com os outros moradores da Ilpi em que residem. Por meio de atividades cotidianas que incidem em ações comunicativas, tais representações indicam a relevância de as Ilpis expandirem atividades coletivas para a boa utilização do tempo livre por parte de seus residentes, resgatando seu papel social e convivência com outros idosos residente na mesma Ilpi (SIMEAO et al., 2018).

Corroborando os resultados deste estudo qualitativo, outras pesquisas indicam que a participação dos idosos na comunidade ajuda a afastar a solidão, contribuindo para um envelhecimento ativo, que pode influenciar positivamente na sua qualidade de vida e como sentimento de pertencimento e fortalecimento de laços afetivos (YARCE-PINZÓN; HIDALGO-SOTELO; NARVÁEZ-CEBALLOS, 2018; FIGUEIREDO, 2016).

O fato de pertencer a um determinado grupo é determinante para que a vida institucionalizada não seja sinônimo de isolamento e exclusão social. De acordo com a Organização Mundial da Saúde, a inatividade e o apoio social inadequados estão associados, não apenas a um aumento de mortalidade, morbidade e problemas psicológicos, mas, também, de um abatimento na saúde e bem-estar em geral, pois a ruptura de laços pessoais e a solidão estão coadunadas a um declínio de saúde tanto física quanto mental (OMS, 2005a).

O trabalho dialógico realizado com idosos institucionalizados, participantes deste estudo, levou-os a preencher um vazio, vencendo a rotina cotidiana da instituição, conforme eles próprios enunciaram. Assim, conseguem colocar-se em movimento e vencer a regularidade de suas ações diárias e previamente estabelecidas, bem como a ociosidade imposta pelo cumprimento das regras institucionais. Constatou-se que no imaginário dos participantes eles anseiam por participação social, pela valorização e pelo convívio com os outros residentes. Nesse sentido, percebe-se que, ao se oferecer atividades de promoção de saúde, perpassadas pelo diálogo, viabiliza-se a ressignificação de valores negativos vinculados ao processo de institucionalização e de estereótipos associados à velhice.

\section{Classe 3}

As representações sociais dos participantes idosos, identificadas na classe 3 , mostram que nunca é tarde para cuidar da saúde, posto que as manifestações linguísticas dos idosos, retratadas nessa classe temática, evidenciam a clara compreensão representacional para os idosos de que é possível cuidar da própria saúde e realizar autocuidado no ambiente institucionalizado. Dessa forma, pode-se inferir que as representações sociais dos participantes deste estudo confirmam que a organização dos grupos de promoção à saúde constitui uma estratégia capaz de contribuir com a promoção da saúde e autocuidado.

O estímulo e a orientação para o autocuidado aos indivíduos idosos são condutas primordiais para se assegurar que eles permaneçam autônomos e independentes pelo período mais longo possível, para que o idoso viva uma velhice desprendida de cuidado formal ou informal realizado por outrem. 
Tratando-se do tema acerca do caráter transformador da educação para o autocuidado aos idosos, este leva-os ao envelhecimento bem-sucedido na medida em que se promove flexibilidade cognitiva e ajustamento social (WHO, 2005a). Abordar o tema da saúde junto aos idosos institucionalizados representa um desafio quando pensado pela ótica da promoção da saúde e do envelhecimento ativo, tendo em vista que os idosos, em sua maioria, apresentam mais susceptibilidade para doenças de fundo orgânicas e psíquicas, levando-os a uma fragilidade e perda da autonomia, o que não favorece um envelhecimento bem-sucedido. As possibilidades e limites das práticas educativas no âmbito das Ilpis configuram uma alternativa capaz de promover saúde e (re)significar a vida dos idosos residentes, empoderando-os e levando-os a se reconhecerem como sujeitos ativos na Ilpi, com inúmeras possibilidades de adquirirem novos conhecimentos a partir das interações dialógicas.

Nesta classe configura-se a relevância das atividades dialógicas para a promoção da saúde, assegurando a essa população a possibilidade de fazer escolhas mais saudáveis dando e mantendo a sua autonomia ao longo do ciclo vital. As ações de educação em saúde, voltadas à pessoa idosa, são ferramentas importantes para a tomada de consciência e mudança de comportamentos passíveis de favorecer sua qualidade de vida, contribuindo para que o idoso seja protagonista do seu próprio cuidado (WHO, 2005b; CLARES; BORGES; FREITAS, 2017; DIAS et al., 2017), pois o autocuidado é considerado um promotor e, também, um recurso importante para a prevenção de complicações de ordem orgânica (ALMEIDA; OLIVEIRA, 2017), contribuindo positivamente para a conservação da independência entre os idosos institucionalizados (IMAGINÁRIO, 2017).

As atuais políticas voltadas a atender a população idosa preconizam o envelhecimento ativo, assistindo a todas as necessidades de saúde explicitadas na Política Nacional de Saúde da Pessoa Idosa, cujo principal objetivo é aumentar os anos de vida saudável e a máxima capacidade funcional do sujeito que envelhece (BRASIL, 2006). Com esse entendimento, ressalta-se que os idosos institucionalizados demonstram interesses em assuntos que envolvem saúde e autocuidado, desde que seja respeitada a singularidade e seu potencial de aprendizagem.

\section{CONSIDERAÇÕES FINAIS}

A representação social expressa pelos idosos institucionalizados acerca das ações dialógicas compartilhadas nos grupos de promoção à saúde, descor- tina um cenário de reconhecimento e pertencimento dos participantes como sujeitos ativos, integrantes de uma rede relacional com os demais idosos, o que confirma a importância e a necessidade da realização de práticas dialógicas junto as pessoas idosas, institucionalizadas ou não, em razão de promover a ampliação da rede social, privilegiando e propiciando uma meIhor convivência, o fortalecimento e o entrosamento entre essa população, pela singela possibilidade de poderem expressar livremente seus anseios e receios a partir do envolvimento com o grupo de promoção da saúde.

Nesse sentido, percebe-se que, ao se oferecer atividades de promoção de saúde, perpassadas pelo diálogo, além da possibilidade de expansão da rede social, os idosos (re)significam os valores negativos vinculados ao processo de envelhecimento, vencendo estereótipos associados à velhice.

Este estudo restringe-se a compreender e interpretar os achados encontrados em duas Ilpis, sendo esta uma limitação do estudo. Sugere-se o desenvolvimento de novas pesquisas que utilizem essas ou outras perspectivas teóricas e metodológicas e que envolvam um número mais representativo de participantes, tendo em vista a relevância das atividades grupais em Ilpis para a promoção e ampliação da integração, da aproximação e da participação social dos idosos, ajudando-os a serem reconhecidos como sujeitos sociais.

\section{REFERÊNCIAS}

ANDRADE, L. M. B. et al. Perfis de integração social entre idosos institucionalizados não frágeis no município de $\mathrm{Na}$ tal, Rio Grande do Norte. Ciência \& Saúde Coletiva, Rio de Janeiro, set. 2018. Disponível em: http://www.cienciaesaudecoletiva.com.br/artigos/perfis-de-integracao-social-entre-idosos-institucionalizados-nao-frageis-no-municipio-de-natal-rio-grande-do-norte/16956. Acesso em: 8 dez. 2018.

ALMEIDA, L. B.; OLIVEIRA, P. R. H. Autocuidado do idoso: revisão sistemática da literatura. Revista Espacios, Venezuela, v. 38, n. 28, p. 3, 2017.

ALVES, R. Se eu pudesse viver minha vida novamente. 1. ed. São Paulo: Planeta, 2016. 160 p.

BARROS, T. V. P. et al.; Functional capacity of institutionalized elderly people: an integrative review. ABCS Health Sci, 41(3):176-180, 2016. Disponivel em: https://www.portalnepas.org.br/abcshs/article/view/908. Acesso em: 19 set. 2019.

BOSI, E. O tempo vivo da memória: ensaios de psicologia social. 2. ed. São Paulo: Ateliê Editorial, 2003. 219 p.

BOSI, E. Velhos amigos. 5. ed. São Paulo: Companhia das Letras, 2008. $112 \mathrm{p}$. 
BRASIL. Lei no 8.842, de 4 de janeiro de 1994. Dispõe sobre a política nacional do idoso, cria o Conselho Nacional do Idoso e dá outras providências. Diário Oficial da União, Brasília, 1994.

BRASIL. Lei no 10.741, de 1ㅇ de outubro de 2003 (Estatuto do Idoso), e legislação correlata. 2. ed. Brasília: Câmara dos Deputados; Coordenação Edições Câmara, 2003.

BRASIL. Portaria no 2.528 de 19 de outubro de 2006. Aprova a Política Nacional de Saúde da Pessoa Idosa. Diário Oficial da União, Brasília, 2006.

BRASIL. Ministério da Saúde. Conselho Nacional de Saúde. Comissão de ética e Pesquisa (CONEP). Resolução no 466, de 12 de dezembro de 2012. Diário Oficial da República Federativa do Brasil, Brasília, DF, 2012. Disponível em: http:// conselho.saude.gov.br/resolucoes/2012/Reso466.pdf. Acesso em: 14 jul. 2016.

BRASIL. Ministério do Desenvolvimento Social. Encontro sobre integração entre serviços e benefícios socioassistenciais para a pessoa idosa. 28 e 29 de junho de 2018. São Paulo.

CAMARGO, B. V.; JUSTO, A. M. IRAMUTEQ: um software gratuito para análise de dados textuais. Periódicos Eletrônicos em Psicologia, Ribeirão Preto, v. 21, n. 2, p. 513-518, dez. 2013. Disponível em: http://pepsic.bvsalud.org/scielo. php?script=sci_arttext \&pid=S1413-389X2013000200016. Acesso em: 19 set. 2019.

CLARES, J. W. B.; BORGES, C. L.; FREITAS, M. C. Institutionalized older: educational proposal in the freirean perspective. Journal of Nursing Ufpe, v. 11, n. 2, p. 1.073-1.077, Feb. 2017. Disponível em: https://periodicos.ufpe.br/revistas/ revistaenfermagem/search/search?query=Institutionalize$\mathrm{d}+$ older\%3A+educational+proposal+in+the+freirean+perspective \&authors $=\&$ title $=$ \&abstract $=$ \&galleyFullText $=$ \& suppFiles $=\&$ dateFrom Month $=\&$ dateFromDay $=\&$ dateFrom Year $=\&$ dateToMonth $=\&$ dateToDay $=\&$ dateToYear $=\&-$ dateToHour=23\&dateToMinute=59\&dateToSecond=59\&discipline $=\&$ subject $=\&$ type $=\&$ coverage $=\&$ indexTerms. Acesso em: 19 set. 2019

DIAS, E. G. et al. Desafios da prática do autocuidado do idoso portador de diabetes mellitus tipo 2. Revista de Saúde e Educação-Sustinere, v. 5, n. 1, p. 38-53, 2017.

FIGUEIREDO, M. L. G. Participação social e redes sociais pessoais de idosos. 2016. Dissertação (Mestrado em Psicologia Clínica) - Instituto Superior Miguel Torga - ISMT, Coimbra, 2016.

IMAGINÁRIO, C. et al. Atividades de vida diária e estilos de autocuidado em idosos institucionalizados. In: CONGRESSO INTERNACIONAL DE INVESTIGAÇÃO, INOVAÇÃO \& DESENVOLVIMENTO EM ENFERMAGEM - Nursid. Livro de Resumos. Escola Superior de Enfermagem do Porto, jul. 2017. p. 119.

IBGE. Instituto Brasileiro de Geografia e Estatística. Síntese de indicadores sociais: uma análise das condições de vida da população brasileira - 2016. Rio de Janeiro: IBGE, 2016. Disponível em: http://biblioteca.ibge.gov.br/visualizacao/ livros/liv98965.pdf. Acesso em: 23 mar. 2017.
MANTOVANI, E. P.; LUCCA, S. R.; NERI, A. L. Associações entre significados de velhice e bem-estar subjetivo indicado por satisfação em idosos. Revista Brasileira de Geriatria e Gerontologia, v. 19, n. 2, p. 203-222, 2016.

MARTINS, N. I. M. et al. Instrumentos de avaliação cognitiva utilizados nos últimos cinco anos em idosos brasileiros. Ciência \& Saúde Coletiva, Rio de Janeiro, v. 24, n. 7, p. 2.513-2.530, 2019.

MAYORALAS, G. F. et al. Active ageing and quality of life: factors associated with participation in leisure activities among institutionalized older adults, with and without dementia. Aging Ment Health, v. 19, n. 11, p. 1.031-1.041, 2015.

MINAYO, M. C. S.; GUALHANO, L. Problemas de saúde e vulnerabilidade da população idosa [on-line]. SciELO em Perspectiva/Press Releases, 2017. Disponível em: https:// pressreleases.scielo.org/blog/2017/01/03/problemas-de-saude-e-vulnerabilidade-da-populacao-idosa. Acesso em: 11 fev. 2019.

MOSCOVICl, S. Representações sociais: investigações em psicologia social. 9. ed. Petrópolis: Vozes, 2012.

NASCIMENTO, M. E. B. Representação social dos cuidados à saúde de pessoas com doença renal crônica: elementos para autogestão. 2016. Tese (Doutorado em Enfermagem) -Universidade Federal do Paraná, Curitiba, 2016.

OMS. Organização Mundial da Saúde. Envelhecimento ativo: uma política de saúde. Contribuição da Organização Mundial da Saúde para o segundo encontro mundial sobre envelhecimento, realizada em Madrid, 2002. Tradução Organização Pan-Americana de Saúde, Brasília, 2005.

OMS. Organización Mundial de la Salud. ASAMBLEA MUNDIAL DE LA SALUD, 69.,. Punto 13.4 del orden del día provisional. Genebra, 2016.

SIMEAO, S. F. A. P. et al. Estudo comparativo da qualidade de vida de idosos asilados e frequentadores do centro dia. Ciênc. Saúde Coletiva, Rio de Janeiro, v. 23, n. 11, p. 3.9233.934, nov. 2018.

WHO. World Health Organization. Envelhecimento ativo: uma política de saúde. Brasília, DF: Organização Pan-Americana de Saúde, 2005a.

WHO. World Health Organization. The Bangkok Charter for Health Promotionin a Globalized World. Bangkok, 11, Aug. 2005b. Disponível em: http://www.who.int/healthpromotion/conferences/6gchp/bangkok_charter/en/. Acesso em: 23 jul. 2016.

WHO. World Health Organization. World report on Ageing and Health. Geneve: WHO Library, 2015.

YARCE-PINZÓN, E.; HIDALGO-SOTELO, Y. G.; NARVÁEZ-CEBALLOS, R. Participación social de un grupo de adultos mayores del corregimiento de Obonuco. $R U$, v. 36, n. 1, p. 14, 2018. 